\title{
A Phenomenological Theory of Gravitation Based on a Generalization of the Special Relativistic Energy Aspect
}

Frank Spieweck

\author{
Z. Naturforsch. 40 a, 173-182 (1985); received October 17, 1984
}

The special relativistic time dilatation and length contraction formulae are written in a general form containing only the ratio of the total energy and the rest energy. Then, it is assumed that the total energy may not only consist of the rest energy and the translational energy but also of the rest energy and potential or rotational energy.

Restricted transformations are given, describing the transition between local systems of reference; in this case, the Euclidean geometry is needed only.

The dependencies of frequency $v$, velocity of light $c$, and inertial mass $m$ on the scalar gravitational potential $U$,

$$
\begin{aligned}
& v^{\prime}=v\left(1-\Delta U / c^{2}\right) / \sqrt{1-\Delta U^{2} / c^{4}}, \quad c^{\prime}=c\left(1-\Delta U / c^{2}\right) /\left(1+\Delta U / c^{2}\right), \\
& m^{\prime}=m\left[\left(1+\Delta U / c^{2}\right) / \sqrt{1-\Delta U^{2} / c^{4}}\right]^{3},
\end{aligned}
$$

to first order of $\Delta U / c^{2}$, are the same ones as known from the theory of general relativity.

Besides the dependencies of physical quantities on the scalar potential $U$, the dependencies on a gravitational vector potential $\boldsymbol{A}$, e.g., a rotational potential, are given, too.

In an appendix, a gravitational field theory is presented, for which the same phenomenology can be used.

\section{Introduction}

According to the theory of special relativity [1], time and length depend on the mechanical energy: A clock having a higher translational energy runs faster than a clock in a "rest" frame of reference.

Here, the following generalization will be made: Any clock being positioned at a "higher" mechanical potential runs faster than a clock at a "lower" potential; and any measuring-rod shortens at a "lower" potential.

As only local systems of reference shall be considered, the Euclidean geometry is needed, only.

\section{Principle of General Relativity}

The principle of general relativity shall be formulated in the following way. All physical relations, defined with respect to a frame of reference $K(x, y, z, t)$, e.g.

or

$$
W=h v \quad[2]
$$

$$
W=m c^{2} \quad[3],
$$

Reprint requests to Dr. F. Spieweck, Nahestr. 19, D-3300 Braunschweig. where $m$ is the inertial mass, can be formulated in any local inertial or non-inertial frame of reference $K^{\prime}\left(x^{\prime}, y^{\prime}, z^{\prime}, t^{\prime}\right)$, too:

$$
\begin{aligned}
& W^{\prime}=h^{\prime} v^{\prime}, \\
& W^{\prime}=m^{\prime} c^{\prime 2} .
\end{aligned}
$$

It shall be anticipated that there exists a physical law,

$$
h^{\prime}=h \text {. }
$$

Thus, (3) becomes

$$
W^{\prime}=h v^{\prime} .
$$

\section{Restricted Transformations}

The transition from a local system of reference $K$ to another local system $K^{\prime}$ shall be called a restricted transformation. That means, a physical process occurring e.g. at $K(0,0,0, t)$ can be described also from $K^{\prime}$ using physical units of $K^{\prime}$.

\subsection{Restricted Lorentz transformation}

If a physical process consists of neighbouring events, the origin of a local system of reference $K(0,0,0)$ can be placed near the events. 
If two events near

$$
x=0
$$

are being considered, then, the Lorentz transformation

$$
t^{\prime}=\left(t-v \cdot x / c^{2}\right) / \sqrt{1-v^{2} / c^{2}}
$$

reduces to the restricted Lorentz transformation

$$
t^{\prime}=t / \sqrt{1-t^{2} / c^{2}} \text {. }
$$

Similarly, the Lorentzian length contraction turns out to be

$$
\Delta L^{\prime}=\Delta L \sqrt{1-v^{2} / c^{2}} .
$$

Due to (7), $K$ is considered to be a rest frame, and $K^{\prime}$ is called a moving frame of reference. That means, any atom of a clock or of a measuring-rod belonging to the moving frame $K^{\prime}$ has - with respect to the frame $K-$ the total energy

$$
W=W_{\text {tot }}=m c^{2} / \sqrt{1-v^{2} / c^{2}},
$$

whereas, if measured with respect to $K^{\prime}$, it has the energy

$$
W^{\prime}=m^{\prime} c^{\prime 2} \text {. }
$$

Now, it is essential that the physical units of $K$ and $K^{\prime}$ must not be transformed, as was already stated in a previous paper [4]. That means, the rest energy of an atom has always the same numerical value,

$$
m c^{2}=m^{\prime} c^{\prime 2} \text {. }
$$

Therefore, (11) can be also written as

$$
W=W_{\mathrm{tot}}=m^{\prime} c^{\prime 2} / \sqrt{1-v^{2} / c^{2}}
$$

or, due to (4),

$$
W=W^{\prime} / \sqrt{1-r^{2} / c^{2}},
$$

that means

$$
W^{\prime}=W \sqrt{1-v^{2} / c^{2}} .
$$

Dividing (15) by (5) yields the red-shift due to the transversal Doppler effect

$$
v^{\prime}=v \sqrt{1-v^{2} / c^{2}},
$$

and due to

$$
\text { or } \quad \begin{aligned}
& t=1 / v \\
& t^{\prime}=1 / v^{\prime},
\end{aligned}
$$

the time dilatation (9).
Due to (13) and (14), (9) and (10) can be written as

$$
t^{\prime}=t \cdot W_{\text {tot }} / W
$$

and

$$
\Delta L^{\prime}=\Delta L \cdot W / W_{\text {tot }} \quad \text { (see*, p. 182). }
$$

\subsection{Restricted gravitational transformations}

If (19) and (20) are interpreted in a generalized way, $K^{\prime}$ is a system of reference having higher mechanical energy than $K$; that means, clocks and measuring-rods in $K^{\prime}$ have higher mechanical energy than clocks and measuring-rods in $K$.

Whereas, so far, in Chapter 3.1 the total mechanical energy consisted of the rest energy and the translational energy,

$$
W_{\mathrm{tot}} \approx W+m v^{2} / 2,
$$

now, the total mechanical energy shall consist of the rest energy and the general gravitational energy, consisting either of the potential or of the rotational energy.

The potential energy is

$$
W_{\text {pot }}=\int q_{\mathrm{g}} \mathrm{d} U^{*} \approx q_{\mathrm{g}} \Delta U^{*},
$$

where $q_{\mathrm{g}}$ is the gravitational load or mass and $U^{*}$ is the (true) Newtonian potential.

The rotational potential will be

$$
W_{\mathrm{rot}} \approx m r^{2} \Omega^{2} / 2 .
$$

In this paper, the question of the equality of the inertial and gravitational mass will be (mainly) left open (see Chapter 6.3). In this case, it is convenient to introduce the quasi-Newtonian potential

$$
U=U^{*} \cdot q_{\mathrm{g}} / m .
$$

\subsection{Transformations within static gravitational fields}

\subsection{Time dilatation}

In a static gravitational field, (19) may be written as

$$
t^{\prime} \approx t\left(W+W_{\mathrm{pot}}\right) / W,
$$

or, due to (22) and (24)

$$
t^{\prime} \approx t\left(1+\Delta U / c^{2}\right) .
$$

As in the case of the Lorentz transformation [5], the (restricted) transformation (26) can be made reciprocal by introducing a factor $N$,

$$
\begin{aligned}
& t^{\prime}=t\left(1+\Delta U / c^{2}\right) / N, \\
& t=t^{\prime}\left(1+\Delta U^{\prime} / c^{\prime 2}\right) / N .
\end{aligned}
$$


As it shall be

$$
\Delta U^{\prime} / c^{\prime 2}=-\Delta U / c^{2},
$$

(28) becomes

$$
t=t^{\prime}\left(1-\Delta U / c^{2}\right) / N .
$$

Then, from multiplying (27) and (30), $N$ turns out to be

$N=\sqrt{\left(1+\Delta U / c^{2}\right)\left(1-\Delta U / c^{2}\right)}=\sqrt{1-\Delta U^{2} / c^{4}}$.

Thus, the exact time dilatation due to a static gravitational field becomes

$$
t^{\prime}=t\left(1+\Delta U / c^{2}\right) / \sqrt{1-\Delta U^{2} / c^{4}} .
$$

\subsection{Length contraction}

Considering the transformation of length, (20) becomes

$$
\Delta L^{\prime} \approx \Delta L W /\left(W+W_{\text {pot }}\right) .
$$

Then, from similar evaluations as made in the previous chapter 3.211 , the length contraction due to a scalar gravitational field turns out to be

$$
\Delta L^{\prime}=\Delta L\left(1-\Delta U / c^{2}\right) / \sqrt{1-\Delta U^{2} / c^{4}} .
$$

Of course, as $U$ is a scalar, this length contraction does not depend on a direction (e.g. of the gravitational field, as was believed by Einstein in 1916 [6] and by Ferrari [7].

3.213. Transformation of further physical quantities

From (17) and (18), the red-shift due a static gravitational field turns out to be

$$
v^{\prime}=v\left(1-\Delta U / c^{2}\right) / \sqrt{1-\Delta U^{2} / c^{4}} .
$$

Due to (22) and (24), (35) can be also written as

$$
\begin{aligned}
v^{\prime} & \approx v-v \Delta U / c^{2}=v-v m \Delta U / m c^{2} \\
& =v-v m \Delta U / h v,
\end{aligned}
$$

i.e.

$$
v^{\prime}=v-W_{\mathrm{pot}} / h .
$$

Here, the Planck constant $h$ plays a similar fundamental role as the velocity of light in the Lorentz transformation. Thus, the assumption

$$
h^{\prime}=h
$$

seems to be justified.

From (5) and (35), the transformation of energy within a static gravitational field turns out to be

$$
W^{\prime}=W\left(1-\Delta U / c^{2}\right) / \sqrt{1-\Delta U^{2} / c^{4}} .
$$

From (32) and (34), the transformation of velocity becomes

$$
r^{\prime}=v\left(1-\Delta U / c^{2}\right) /\left(1+\Delta U / c^{2}\right) .
$$

And, as $r$ can be an arbitrary velocity, the transformation of the velocity of light, as well, within a static gravitational field results to be

$$
\text { or } \quad \begin{aligned}
c^{\prime} & =c\left(1-\Delta U / c^{2}\right) /\left(1+\Delta U / c^{2}\right), \\
c^{\prime} & \approx c\left(1-2 \Delta U / c^{2}\right) .
\end{aligned}
$$

That means, (34) yields the experimental value of the light bending [8], whereas the Galilean transformation

$$
\Delta L^{\prime}=\Delta L
$$

would lead to only half the light deflection, as in the early Einsteinian theory of 1911 [9].

From (2), (4), (38), and (40), the increase of the inertial mass within a static gravitational field becomes

$$
\begin{aligned}
& m^{\prime}=m\left[\left(1+\Delta U / c^{2}\right) / \sqrt{1-\Delta U^{2} / c^{4}}\right]^{3}, \\
& m^{\prime} \approx m\left(1+3 \Delta U / c^{2}\right),
\end{aligned}
$$

which is the experimental result of the perihelion advance of mercury [8].

Due to (39) and (43), the momentum

$$
p=m v
$$

will transform as

$$
\text { or } \quad \begin{aligned}
p^{\prime} & =m^{\prime} v^{\prime}, \\
p^{\prime} & =p\left(1+\Delta U / c^{2}\right) / \sqrt{1-\Delta U^{2} / c^{4}} .
\end{aligned}
$$

In addition to (5), the electric charge shall be assumed to be invariant, too,

$$
q^{\prime}=q .
$$

Thus, the electric current

$$
I=q / t,
$$

according to (32) and (48), will transform as

$$
\text { i.e. } \quad \begin{aligned}
& I^{\prime}=q / t^{\prime}, \\
& I^{\prime}=I\left(1-\Delta U / c^{2}\right) / \sqrt{1-\Delta U^{2} / c^{4}} .
\end{aligned}
$$

And an electrical voltage

$$
V=W / q
$$

will transform as

$$
\text { or } \quad \begin{aligned}
& V^{\prime}=W^{\prime} / q^{\prime}, \\
& V^{\prime}=V\left(1-\Delta U / c^{2}\right) / \sqrt{1-\Delta U^{2} / c^{4}} .
\end{aligned}
$$




\subsection{Invariants}

As the unit of action, $h$, is invariant against a transition within a static gravitational field, action itself must be invariant, too.

Indeed, according to (32) and (38), it is

$$
\begin{aligned}
W^{\prime} t^{\prime}= & W\left(1-\Delta U / c^{2}\right) / \sqrt{1-\Delta U^{2} / c^{4}} \\
& \cdot t\left(1+\Delta U / c^{2}\right) / \sqrt{1-\Delta U^{2} / c^{4}}=W t,(5)
\end{aligned}
$$

and, due to (34) and (47), it results

$$
\begin{aligned}
p^{\prime} \Delta L^{\prime}= & p\left(1+\Delta U / c^{2}\right) / \sqrt{1-\Delta U^{2} / c^{4}} \\
& \cdot \Delta L\left(1-\Delta U / c^{2}\right) / \sqrt{1-\Delta U^{2} / c^{4}}=p \cdot \Delta L,(56)
\end{aligned}
$$

Due to (34), (38), and (40), another mechanical invariant turns out to be $q_{g}^{2} G / m^{2} c^{4}$, see Chapt. 6.3 and [8].

The electrical resistance results from (51) and (54) to be

$$
R^{\prime}=V^{\prime} / I^{\prime}=V / I=R .
$$

Therefore, the vacuum impedance, too, must be an invariant:

$$
z^{\prime}=\left(\mu_{0}^{\prime} / \varepsilon_{0}^{\prime}\right)^{1 / 2}=\left(\mu_{0} / \varepsilon_{0}\right)^{1 / 2}=z .
$$

That means, the vacuum impedance plays a fundamental role in physics, as was already stated by Seifert [10]. According to (58), the fine structure constant [10]

$$
\alpha=z e / 2 h,
$$

of course, is also an invariant:

$$
\alpha^{\prime}=z^{\prime} e^{\prime} / 2 h^{\prime}=z e / 2 h=\alpha .
$$

From (58) and the Maxwellian relation

$$
\text { or } \begin{aligned}
\varepsilon_{0} \mu_{0} & =1 / c^{2} \\
\varepsilon_{0}^{\prime} \mu_{0}^{\prime} & =1 / c^{\prime 2},
\end{aligned}
$$

it results

and

$$
\varepsilon_{0}^{\prime}=\varepsilon_{0}\left(1+\Delta U / c^{2}\right) /\left(1-\Delta U / c^{2}\right)
$$

$$
\mu_{0}^{\prime}=\mu_{0}\left(1+\Delta U / c^{2}\right) /\left(1-\Delta U / c^{2}\right) .
$$

From (34) it can be concluded that the partial derivatives performed in $K^{\prime}$ will be

$$
\operatorname{curl}^{\prime}=\operatorname{curl}\left(1+\Delta U / c^{2}\right) / \sqrt{1-\Delta U^{2} / c^{4}}
$$

and

$$
\operatorname{div}^{\prime}=\operatorname{div}\left(1+\Delta U / c^{2}\right) / \sqrt{1-\Delta U^{2} / c^{4}} .
$$

Therefore, the Maxwellian equations are invariant against a transition within a static gravitational field:

$$
\begin{aligned}
& \operatorname{curl}^{\prime} \boldsymbol{H}^{\prime}=\boldsymbol{j}^{\prime}+\partial \boldsymbol{D}^{\prime} / \partial t^{\prime} \\
& \operatorname{curl}^{\prime} \boldsymbol{E}^{\prime}=-\partial \boldsymbol{B}^{\prime} / \partial t^{\prime} \\
& \operatorname{div}^{\prime} \boldsymbol{D}^{\prime}=\varrho^{\prime} \\
& \operatorname{div}^{\prime} \boldsymbol{B}^{\prime}=0
\end{aligned}
$$

That means, electromagnetic processes taking place near the origin of the system of reference $K$, can be also described from the system of reference $K^{\prime}$, using physical quantities of $K^{\prime}$.

\subsection{Transformations within} dynamical gravitational fields

In a rotating system of reference, e.g. on earth, the atoms of a clock in an outer local system of reference $K$ have a lower total mechanical energy than the atoms of a clock in an inner local system of reference $K^{\prime}$ (e.g. at $r=0$ ), as the work

$$
W_{\mathrm{rot}} \approx m r^{2} \Omega^{2} / 2
$$

must be done, if a clock is transported from the origin of $K$ to the origin of $K^{\prime}$.

Now, in a dynamical - i.e. rotational - gravitational field, (19) can be written as

$$
t^{\prime} \approx t \cdot\left(W+W_{\mathrm{rot}}\right) / W,
$$

or, according to (23),

$$
t^{\prime} \approx t\left(1+r^{2} \Omega^{2} / 2 c^{2}\right) \text {. }
$$

Similarly, (20) becomes

$$
\Delta L^{\prime} \approx \Delta L\left(1-r^{2} \Omega^{2} / 2 c^{2}\right) \text {. }
$$

Thus, obviously, the exact restricted gravitational transformations within a rotational field should be

and

$$
t^{\prime}=t / \sqrt{1-r^{2} \Omega^{2} / c^{2}}
$$

$$
\Delta L^{\prime}=\Delta L \sqrt{1-r^{2} \Omega^{2} / c^{2}} \text {. }
$$

Using the theory of general relativity [11], Landau and Lifschitz derive a similar expression, when they were considering the ratio of the circumference and the radius of a circle in a rotating system; they obtain [12]

$$
2 \pi / \sqrt{1-\Omega^{2} r^{2} / c^{2}}
$$

As (74) and (75) are similar transformations as the restricted Lorentz transformations (9) and (10), all physical quantities will be correspondingly trans- 
formed, and similar invariants can be calculated as known from the theory of special relativity. Of course, e.g., the Maxwellian equations are invariant against a transition from one rotational system, $K$, to another one, $K^{\prime}$.

\subsection{Combined transformations}

Now, the influence of a static gravitational field on physical quantities shall be combined with the influence of a translational motion or with the influence of a rotational dynamical gravitational field.

3.231. Transition in a static gravitational field combined with a translational motion

E.g., combining the special relativistic Doppler shift with the red-shift due to a static gravitational field (35), yields the total red-shift

$$
v_{-}^{\prime}=v \sqrt{\frac{1-v / c}{1+v / c}} \cdot \frac{1-\Delta U / c^{2}}{\sqrt{1-\Delta U^{2} / c^{4}}} .
$$

3.232. Transition in a static gravitational field combined with a rotational motion

If two rotating systems of reference, $K_{1}$ and $K_{2}$, are being considered, it may be, due to (74),

$$
t^{\prime}=t_{1} / \sqrt{1-r^{2} \Omega^{2} / c^{2}},
$$

as well as

$$
t^{\prime}=t_{2} / \sqrt{1-(\boldsymbol{r}+\boldsymbol{H})^{2}(\boldsymbol{\Omega} \pm \omega)^{2} / c^{2}} .
$$

Therefore, the transition from $K_{1}$ to $K_{2}$ will be

$t_{2}=t_{1} \sqrt{1-(\boldsymbol{r}+\boldsymbol{H})^{2}(\boldsymbol{\Omega} \pm \boldsymbol{\omega})^{2} / c^{2}} / \sqrt{1-r^{2} \Omega^{2} / c^{2}}$.

On earth it is

$$
\Delta U \approx g H,
$$

where it is

$$
g=G M / r^{2}
$$

and $H$ is the difference in height.

Thus, according to (32), the total gravitational transformation turns out to be

$$
\begin{aligned}
t_{2}= & t_{1} \frac{1+g H / c^{2}}{\sqrt{1-g^{2} H^{2} / c^{4}}} \\
& \frac{\sqrt{1-(\boldsymbol{r}+\boldsymbol{H})^{2}(\boldsymbol{\Omega} \pm \omega)^{2} / c^{2}}}{\sqrt{1-r^{2} \Omega^{2} / c^{2}}} .
\end{aligned}
$$

E.g., in the experiment of Hafele and Keating [13], the velocity of the airplane is

$$
\pm v= \pm(r \times \omega) \text {. }
$$

Then, for low heights, from (83) and (84), the usual result $[14,15]$, is obtained:

$$
t_{2} \approx t_{1}\left(1+g H / c^{2} \mp r \Omega v / c^{2}-v^{2} / 2 c^{2}\right) .
$$

\section{Discussion}

\subsection{Verification by experiments}

As the static gravitational term $\Delta U / c^{2}$ usually has a very small value only, e.g.

$$
\Delta U / c^{2} \approx 10^{-6}
$$

(and the rotational term $r^{2} \Omega^{2} / c^{2}$ is even lower) all predictions made by different gravitational theories can be verified only up to first order of $\Delta U / c^{2}$. That means, different formulae, e.g.

$$
\begin{aligned}
& t^{\prime}=t\left(1+\Delta U / c^{2}\right) / \sqrt{1-\Delta U^{2} / c^{4}} \\
& t^{\prime}=t / \sqrt{1-2 \Delta U / c^{2}} \\
& t^{\prime}=t /\left(1-\Delta U / c^{2}\right)
\end{aligned}
$$

yield the same approximation

$$
t^{\prime} \approx t\left(1+\Delta U / c^{2}\right) .
$$

The gravitational red-shift can be tested only in the form

$$
v^{\prime} \approx v\left(1-\Delta U / c^{2}\right)
$$

an observation of the deflection of light yields [8]

$$
c^{\prime} \approx c\left(1-2 \Delta U / c^{2}\right),
$$

and the advance of the perihelion of mercury corresponds to [8]

$$
m^{\prime} \approx m\left(1+3 \Delta U / c^{2}\right) .
$$

\subsection{Other gravitational theories}

The above results, (36), (41), and (44), are obtained also in the gravitational theories of Einstein $[6,11]$, Brans and Dicke [16], Birkhoff [17], Whitehead [18] and Ferrari [7].

An analysis made by Will [19] has shown that the theories of Birkhoff and Whitehead can be ruled out as some of their predictions do not agree with experiments.

As the theory of Brans and Dicke contain adjustable parameters, it is not surprising that the results (36), (41), and (44) can be derived from this theory. 
The considerations presented in this paper use only two assumptions, namely the principle of general relativity (see chapter 2 ) and the equivalence of $\Delta U / c^{2}$ and $\Delta U^{\prime} / c^{\prime 2}$ (see chapter 3.211), (29), which is a more general relation than $m=q_{g}$, - whereas the theory of general relativity uses several assumptions about a metric tensor, see e.g. [11].

Comparing the ideas of the theory of general relativity with the concept established here, the principal difference turns out to be the question of geometry. Whereas in the theory of general relativity the invariant

$$
\mathrm{d} s=\sqrt{g_{\mu v} \mathrm{~d} x_{\mu} \mathrm{d} x_{v}}
$$

is defined to be a "space-time" interval, here, the space-time interval would be conventionally defined as

$$
\text { or } \begin{aligned}
& \sqrt{c^{2} \mathrm{~d} t^{2}-\mathrm{d} L^{2}} \\
& \sqrt{c^{\prime 2} \mathrm{~d} t^{\prime 2}-\mathrm{d} L^{\prime 2}} .
\end{aligned}
$$

Of course, according to this - usual - kind of definition, the space-time interval is not an invariant, as it is - due to (32), (34), and (40)

$$
c^{\prime 2} \mathrm{~d} t^{\prime 2}-\mathrm{d} L^{\prime 2} \neq c^{2} \mathrm{~d} t^{2}-\mathrm{d} L^{2} .
$$

The advantage of the conventional definition of the space-time interval is not only the possibility of further using the Euclidean geometry but also of maintaining the usual sense of all physical quantities, e.g., of energy and momentum.

\subsection{Three transformations - three stages of physics}

In a first stage of physics, classical mechanics is governed by the idea of a universal time

$$
t^{\prime}=t
$$

and a universal space; due to the Galilean transformation it is

$$
\Delta L^{\prime}=\Delta L .
$$

In a second stage of physics, considering electromagnetic processes, time and space become related physical quantities. The idea of space and time is contained in the Lorentz transformation; not $\Delta T$ or $\Delta L$ but only the space-time interval

$$
\sqrt{c^{2} \mathrm{~d} t^{2}-\mathrm{d} L^{2}}
$$

is an invariant.
In a third stage of physics, the measuring process plays the decisive role; here, the Planck constant $h$ governs the uncertainty relations in quantum mechanics and the relations used in this paper, namely

$$
\begin{aligned}
W & =h v \\
h^{\prime} & =h .
\end{aligned}
$$

Whereas in quantum mechanics the kind of measurement is most important, in the gravitational physics considered here, the location where the measurement is being performed must be additionally known. E.g., an experimental result presented as

$$
\Delta t=3 \text { seconds }
$$

is worthless unless it is stated where the measurement has been made (that means, either within the local system of reference $K$ or in the local system of reference $K^{\prime}$ ).

In the third stage of physics neither $\Delta t$ or $\Delta L$ nor $\sqrt{c^{2} \mathrm{~d} t^{2}-\mathrm{d} L^{2}}$ are invariant against a gravitational transformation, but only (- or mainly -) action, i.e. the product of energy and time, $W t$, or of momentum and length, $p \Delta L$.

\section{Conclusion}

The concept of this paper is based upon two main ideas, a generalization of the special relativistic energy aspect and a restriction to local frames of reference.

In a system of higher mechanical energy, irrespective of the kind of energy (gravitational potential energy, translational or rotational kinetical energy), the atomic energy is higher than in a system of lower mechanical energy; and thus, due to the invariance of the Planck constant $h$, the atomic frequencies are higher. Therefore a clock runs faster than a similar clock being positioned in a system of lower mechanical energy. And a similar generalization was made concerning the length contraction.

The physical concept of space and time, similarly as in classical mechanics and in the theory of special relativity, could be derived from a transformation, again, whereas in the theory of general relativity instead of a new transformation a new kind of geometry had been used.

Here, the restriction from extended to local frames of reference opened the possibility to main- 
tain the Euclidean geometry and to preserve the usual meaning of all physical quantities.

Universal or truly fundamental constants, that means invariants against all considered transformations, turned out to be $h, e, z, \alpha$ and the combined constants $\mu_{0} c, \varepsilon_{0} c$ (and, of course, $h / e$ and $h / e^{2}$ ), as well as $q_{\mathrm{g}}^{2} G / \mathrm{m}^{2} c^{4}$, see Chapt. 6.3 and [8].

So far, a phenomenological theory has been given, explaining the dependencies of the main physical quantities on different types of mechanical energy. In an appendix, a simple field theory of gravitation will be developed.

\section{Appendix: A Vector Theory of Gravitation}

Here, again, local frames of reference shall be considered, only. In this case, the Euclidean geometry can be used; that means, a vector theory of gravitation can be built up.

In the above presented concept of a gravitational theory, besides a scalar gravitational potential $U$, it was used also a vector potential, namely $(\boldsymbol{r} \times \boldsymbol{\Omega})$.

Thirring $[20,21]$ first verified that the theory of general relativity - to first order - contains a vector potential $\boldsymbol{A}$, see [11].

In Hund's model theory of general relativity $[5,22]$, a vector potential is defined as

$$
\boldsymbol{A}=-\boldsymbol{v}-\overline{\boldsymbol{\Omega}} \times \boldsymbol{r} .
$$

\subsection{General formalism}

In a vector theory (of gravitation) the MaxwellLorentz formalism can be widely used. But, due to the fact that the Newtonian force is attractive, most gravitational terms have the opposite sign as in the electromagnetic case; this behaviour was already mentioned by Thirring $[20,21]$.

A corresponding formalism was given by the author in a previous paper by introducing a negative field constant [23],

$$
\varepsilon_{\mathrm{g} 0}=-1 / 4 \pi G
$$

Then, due to the "Maxwellian" relation

$$
\varepsilon_{\mathrm{g} 0} \mu_{\mathrm{g} 0}=1 / c^{2},
$$

the second gravitational constant, too, turns out to be negative:

$$
\mu_{\mathrm{g} 0}=-4 \pi G / c^{2}=-x / 2,
$$

where $x$ is the Einsteinian gravitational constant.
Thus, instead of the Newtonian scalar potential $U^{*}$, the - negative - scalar potential

$$
\varphi_{\mathrm{g}}=\frac{1}{4 \pi \varepsilon_{\mathrm{g} 0}} \int \frac{\varrho_{\mathrm{g}} \mathrm{d} V}{r}=-U^{*}
$$

can be defined; and moving mass(densiti)es will give rise to a gravitational vector potential

$$
\boldsymbol{A}_{\mathrm{g}}=\frac{\mu_{\mathrm{g} 0}}{4 \pi} \int \frac{\varrho_{\mathrm{g}} \boldsymbol{v} \mathrm{d} V}{r} .
$$

Then, the polar gravitational field strength becomes

$$
\boldsymbol{E}_{\mathrm{g}}=-\operatorname{grad} \varphi_{\mathrm{g}}-\dot{\boldsymbol{A}}_{\mathrm{g}},
$$

where the second term includes the centrifugal field strength; and the axial gravitational - or Coriolis field strength will be

$$
\boldsymbol{B}_{\mathrm{g}}=\operatorname{curl} \boldsymbol{A}_{\mathrm{g}} \text {. }
$$

Therefore, the gravitational force acting on a test mass $q_{\mathrm{g}}$ is

$$
\boldsymbol{F}=q_{\mathrm{g}}\left(\boldsymbol{E}_{\mathrm{g}}+\boldsymbol{v} \times \boldsymbol{B}_{\mathrm{g}}\right) .
$$

\subsection{Gravitational field equations}

By introducing the exciting vectors $\boldsymbol{D}_{\mathrm{g}}$ and $\boldsymbol{H}_{\mathrm{g}}$ via the relations

and $\begin{aligned} & \boldsymbol{D}_{\mathrm{g}}=\varepsilon_{\mathrm{g} 0} \boldsymbol{E}_{\mathrm{g}} \\ & \boldsymbol{B}_{\mathrm{g}}=\mu_{\mathrm{g} 0} \boldsymbol{H}_{\mathrm{g}},\end{aligned}$

the gravitational field equations will be

$$
\begin{aligned}
& \operatorname{curl} \boldsymbol{H}_{\mathrm{g}}=\boldsymbol{j}_{\mathrm{g}}+\dot{\boldsymbol{D}}_{\mathrm{g}}, \\
& \operatorname{curl} \boldsymbol{E}_{\mathrm{g}}=-\dot{\boldsymbol{B}}_{\mathrm{g}}, \\
& \operatorname{div} \boldsymbol{D}_{\mathrm{g}}=\varrho_{\mathrm{g}}, \\
& \operatorname{div} \boldsymbol{B}_{\mathrm{g}}=0 .
\end{aligned}
$$

In (106), $\boldsymbol{j}_{\mathrm{g}}$ is the total flux of "mass", consisting of the (usual, positive) mass flux $\boldsymbol{j}_{+}$and a negative contribution due to the energy flux $\boldsymbol{S}_{\mathrm{g}}$,

$$
\boldsymbol{j}_{-}=\boldsymbol{S}_{\mathrm{g}} / c^{2}=\left(\boldsymbol{E}_{\mathrm{g}} \times \boldsymbol{H}_{\mathrm{g}}\right) / c^{2}
$$

that means, it is

$$
\boldsymbol{j}_{\mathrm{g}}=\boldsymbol{j}_{+}+\boldsymbol{j}_{-} .
$$

Similarly, in (108), $\varrho_{\mathrm{g}}$ is the total "mass" density, consisting of the (usual, positive) mass density $\varrho_{+}$ and the negative energetical contribution

$$
\varrho_{-}=\left(\boldsymbol{E}_{\mathrm{g}} \boldsymbol{D}_{\mathrm{g}}+\boldsymbol{H}_{\mathrm{g}} \boldsymbol{B}_{\mathrm{g}}\right) / 2 c^{2},
$$

thus it is

$$
\varrho_{\mathrm{g}}=\varrho_{+}+\varrho_{-} .
$$


Then, i.e. combining the equations (111) and (113) with the field equations (106) to (109), the gravitational field equations can be also written in the manner done by Hund [5, 22].

As the Maxwellian equations themselves (67)-(70), the gravitational field equations (106) till (109), too, are invariant against a gravitational transformation.

\subsection{Equations of motion}

Using the Lagrangean function [23]

$$
L=-m c^{2} \sqrt{1-v^{2} / c^{2}}-q_{\mathrm{g}}\left(\varphi_{\mathrm{g}}-A_{\mathrm{g}} v\right),
$$

the gravitational equations of motion can be derived from the Lagrangean equations

$$
\frac{\mathrm{d}}{\mathrm{d} t}\left(\frac{\partial L}{\partial \boldsymbol{v}}\right)-\frac{\partial L}{\partial \boldsymbol{r}}=0 \text {. }
$$

Thus, the gravitational equations of motion are

$$
\frac{\mathrm{d}}{\mathrm{d} t}\left(\frac{m v}{\sqrt{1-v^{2} / c^{2}}}\right)=q_{\mathrm{g}}\left(\boldsymbol{E}_{\mathrm{g}}+\boldsymbol{v} \times \boldsymbol{B}_{\mathrm{g}}\right) .
$$

\subsection{The general gravitational "field" in a non-inertial frame of reference}

According to the ideas of Hund [5, 22] and Joos [24], the forces caused by the relative motion of the earth and the whole universe can be described in two equivalent ways, the usual one and the following one: The relative motion of the whole universe against a non-inertial frame of reference will give rise to the vector potential (see [22])

$$
\boldsymbol{A}_{\mathrm{g}}=\frac{m}{q_{\mathrm{g}}}(-\boldsymbol{v}-\boldsymbol{\Omega} \times \boldsymbol{r}),
$$

where $v$ and $\boldsymbol{\Omega}$ represent the linear and the rotational motion of the whole universe against the considered local (non-inertial) frame of reference.

If $\boldsymbol{r}$, contrary to Hund's model theory [22], is not considered to be constant $(\dot{\boldsymbol{r}}=0)$ but time dependent as

$$
\dot{r}=\boldsymbol{r} \times \boldsymbol{\Omega},
$$

then, the centrifugal field strength results from the second term of (117) rather than from an - artificial - second scalar potential as in Hund's model theory [22].

According to (101) and (117), the polar gravitational field strength becomes

$$
\boldsymbol{E}_{\mathrm{g}}=\operatorname{grad} U^{*}+\frac{m}{q_{\mathrm{g}}}(\dot{\boldsymbol{v}}+\dot{\boldsymbol{\Omega}} \times \boldsymbol{r}+\boldsymbol{\Omega} \times \dot{\boldsymbol{r}}),
$$

or, due to (24), respectively

$$
\begin{aligned}
& U^{*}=U m / q_{\mathrm{g}}, \\
& \boldsymbol{E}_{\mathrm{g}}=\frac{m}{q_{\mathrm{g}}}\left(\operatorname{grad} U+\dot{\boldsymbol{v}}+\dot{\boldsymbol{\Omega}} \times \boldsymbol{r}-\boldsymbol{\Omega}^{2} \boldsymbol{r}\right) .
\end{aligned}
$$

And the axial gravitational field strength, i.e. the Coriolis field strength, results from (102) and (117) to be

$$
\boldsymbol{B}_{\mathrm{g}}=\frac{m}{q_{\mathrm{g}}} \cdot 2 \boldsymbol{\Omega} .
$$

Thus, according to (103) or (116), the total - or general - gravitational force becomes

$\boldsymbol{F}=m\left(\operatorname{grad} U+\dot{\boldsymbol{v}}+\dot{\boldsymbol{\Omega}} \times \boldsymbol{r}-\Omega^{2} \boldsymbol{r}-2 \boldsymbol{v} \times \boldsymbol{\Omega}\right)$,

which is the same result as known from classical mechanics.

\subsection{Equivalence of inertial and gravitational mass?}

So far, the status of the gravitational mass remained an open question.

Similarly as the Coulomb law

$$
F=\frac{1}{4 \pi \varepsilon_{0}} \cdot \frac{q^{2}}{r^{2}}
$$

contains only the combined items $q^{2} / \varepsilon_{0}$, from the Newtonian law

$$
F=G q_{\mathrm{g}}^{2} / r^{2},
$$

too, only the behaviour of the combination $G q_{\mathrm{g}}^{2}$ can be evaluated, namely

$\left(G q_{\mathrm{g}}^{2}\right)^{\prime}=\left(G q_{\mathrm{g}}^{2}\right)\left(1-\Delta U / c^{2}\right) /\left(1+\Delta U / c^{2}\right)$.

Now, it shall be first emphasized that it will be impossible by the most experiments to decide between the two choices

$$
\begin{array}{ll}
\text { and } & q_{\mathrm{g}}^{\prime}=q_{\mathrm{g}} \\
\text { i.e. } & q_{\mathrm{g}}^{\prime}=q_{\mathrm{g}}\left[\left(1+\Delta U / c^{2}\right) / \sqrt{1-\Delta U^{2} / c^{4}}\right]^{3}, \\
\text { or } \quad & q_{\mathrm{g}}^{\prime}=m^{\prime} \\
& q_{\mathrm{g}}=m,
\end{array}
$$

see also the detailed analysis of Denisov and Logunov [25].

E. g., the period of a mathematical pendulum is

$$
T=2 \pi\left(m L / q_{\mathrm{g}} g\right)^{1 / 2},
$$

where it is

$$
g=G q_{\mathrm{g}} / r^{2} .
$$


Thus, according to (126) it results

$$
T^{\prime}=T\left(1+\Delta U / c^{2}\right) / \sqrt{1-\Delta U^{2} / c^{4}}
$$

that means, concerning the gravitational behaviour, there will be no difference between an atomic clock (due to (32)) and a gravitational pendulum clock (due to (133)).

Second, the gravitational field equations (106) till (109) are invariant against a gravitational transformation, irrespective of the two different choices (127) or (128).

And third, a gravitational fine structure constant, discussed by Cole [26],

$$
\alpha_{\mathrm{g}}=G q_{\mathrm{g}}^{2} \hbar c,
$$

too, only contains the product $G q_{\mathrm{g}}^{2}$. That means, in both cases, $\alpha_{\mathrm{g}}$ will be an invariant,

$$
\alpha_{\mathrm{g}}^{\prime}=\alpha_{\mathrm{g}} \text {. }
$$

\subsection{Equality of inertial}

and gravitational mass?

The Einsteinian choice

$$
q_{\mathrm{g}}=m
$$

has the advantage of being simple, that means, a decision of different types of masses must not be made.

On the other hand, there would not be only differences concerning the general behaviour of electromagnetic and gravitational terms, but, especially the gravitational vacuum impedance $z_{\mathrm{g}}=$ $\left(\mu_{\mathrm{g} 0} / \varepsilon_{\mathrm{g} 0}\right)^{1 / 2}$ would not be an invariant,

$$
z_{\mathrm{g}}^{\prime}=z_{\mathrm{g}}\left(1-\Delta U / c^{2}\right)^{3} /\left(1+\Delta U / c^{2}\right)^{3},
$$

whereas (in Chapt. 3.214) the electromagnetic vacuum impedance $z$ turned out to be invariant against a gravitational transformation. But, perhaps, (136) may indicate that the propagation of gravitational waves will be different from the behaviour of electromagnetic waves.

Of course, a similar behaviour of $z$ and $z_{\mathrm{g}}$ would be obtained, if, contrary to the choice

$$
q^{\prime}=q,
$$

it would be assumed

$$
q^{\prime}=q\left[\left(1+\Delta U / c^{2}\right) / \sqrt{1-\Delta U^{2} / c^{4}}\right]^{3} .
$$

But then, neither $h / e$ nor $h / e^{2}$ remain invariant.

Another - fallacious - argument for the choice (130) seems to be the fact that atoms having higher kinetical energy will also have the higher weight; but weight is not only proportional to $q_{\mathrm{g}}$ or $q_{\mathrm{g}}^{2}$, but to $G q_{\mathrm{g}}^{2}$ !

6.32. Similar behaviour of electric charge and gravitational mass?

The alternative choice,

$$
q_{\mathrm{g}}^{\prime}=q_{\mathrm{g}},
$$

seems to bear more advantages than the Einsteinian choice: First, the gravitational behaviour of the corresponding electromagnetic and gravitational terms will be equivalent; and $h / e$ and $h / e^{2}$ remain invariants.

Second, not only the electromagnetic vacuum impedance $z$, but also the gravitational vacuum impedance $z_{\mathrm{g}}$ will be an invariant,

$$
z_{\mathrm{g}}^{\prime}=z_{\mathrm{g}} \text {. }
$$

\section{Note added in proof:}

Equations (19) and (20) can be easier obtained by multiplying numerator and denominator of (9) and (10) with $m c^{2}$ - taking into account (2) and (11).
[1] A. Einstein, Ann. Phys. (4) 17,891 (1905).

[2] A. Einstein, Ann. Phys. (4) 17, 132 (1905).

[3] A. Einstein, Ann. Phys. (4) 18, 639 (1905).

[4] F. Spieweck, PTB-Mitteilungen 88, 323 (1978).

[5] F. Hund, Theoretische Physik, Bd. II, 3. Aufl., B. G. Teubner-Verlag, Stuttgart 1957.

[6] A. Einstein, Ann. Phys. (4) 49, 769 (1916).

[7] J. A. Ferrari, Nouvo Cim. $\mathbf{7 8}$ B, 53 (1983).

[8] H. Dehnen, H. Hönl, and K. Westpfahl, Ann. Phys. (7) 6, 370 (1960).
[9] A. Einstein, Ann. Phys. (4) 35, 898 (1911).

[10] H. U. Seifert, Ann. Phys. (7) 16, 269 (1965).

[11] A. Einstein, Grundzüge der Relativitätstheorie, Vieweg \& Sohn, Braunschweig 1956.

[12] L. D. Landau und E. M. Lifschitz, Lehrbuch der Theoretischen Physik, Bd. II, 4. Aufl., AkademieVerlag, Berlin 1967.

[13] J. C. Hafele and R. E. Keating, Science 177, 168 (1972).

[14] J. C. Hafele and R. E. Keating, Science 177, 166 (1972). 
[15] R. Schlegel, Am. J. Phys. 42, 183 (1974).

[16] C. Brans and R. H. Dicke, Phys. Rev. 124, 925 (1961).

[17] G. D. Birkhoff, Proc. N.A.S. 29, 231 (1943); 30, 324 (1944).

[18] A. N. Whitehead, The Principle of Relativity, Cambridge University Press 1922.

[19] C. M. Will, Phys. Today (Oct. 1972), 23.

[20] H. Thirring, Phys. Z. 19, 33 (1918).

[21] H. Thirring, Phys. Z. 19, 204 (1918).
[22] F. Hund, Grundbegriffe der Physik, Bibliographisches Institut, Mannheim/Zürich 1969.

[23] F. Spieweck, Astron. Astrophys. 12, 278 (1971).

[24] G. Joos, Lehrbuch der Theoretischen Physik, 13. Aufl., Akademische Verlagsgesellschaft, Wiesbaden 1977.

[25] V. I. Denisov and A. A. Logunov, Sov. J. Part. Nucl. 13, 317 (1982).

[26] K. D. Cole, Nuovo Cim. 26 B, 370 (1975). 